\title{
Evolução do Plano de Ação para Prevenção e Controle do Desmatamento na Amazônia Legal
}

\author{
[ Evolution of the Action Plan for Prevention and Control \\ of Deforestation in the Brazilian Amazon
}

\section{Natália Girão Rodrigues de Mello}

\section{Paulo Artaxo}

\begin{abstract}
RESUMO • O presente estudo analisou a evolução da implementação do Plano de Ação para Prevenção e Controle do Desmatamento na Amazônia Legal (PPCDAm). Para tal, foi realizada análise de: publicações científicas que caracterizam o problema identificado; documentos que compreendem a Fase I (2004-2008), a Fase II (2009-2011) e a Fase III (2012-2015) do PPCDAm; avaliações realizadas sobre a Fase I (realizada por Abdala, 2008) e sobre a Fase II do PPCDAm (realizada por Ipea; GIZ; Cepal, 2011); e impactos do PPDCAm na redução do desmatamento. Conclui-se que o PPCDAm trouxe resultados significativos para a contenção do desmatamento na Amazônia Legal, mas ainda se fazem necessários aprimoramentos para que sejam alcançados os objetivos de promoção de atividades sustentáveis na região. • PALAVRAS-CHAVE・Desmatamento; Amazônia; políticas públicas. • ABSTRACT • The
\end{abstract}

\begin{abstract}
present study examined the evolution of the implementation of the Action Plan for the Prevention and Control of Deforestation in the Brazilian Amazon (PPCDAm). With this purpose, it was performed the analysis of: scientific publications that characterize deforestation in the Brazilian Amazon; documents comprising the Phase I (2004-2008), Phase II (20092011) and Phase III (2012-2015) of the PPCDAm; independent assessments of the Phase I (performed by Abdala, 2008) and the Phase II PPCDAm (performed by Ipea; GIZ; Eclac, 2011); and impacts of the PPCDAm in reducing deforestation. It is concluded that the implementation of the PPCDAm has brought significant results to control the deforestation in the Brazilian Amazon, but improvements aimed at attaining the objectives of the promotion of sustainable activities in the region are still needed. - KEYWORDS - Deforestation; Brazilian Amazon; policies.
\end{abstract}

Recebido em 4 de novembro de 2015

Aprovado em I3 de setembro de 2016

MELLO, Natália Girão Rodrigues de; ARTAXO, Paulo. Evolução do Plano de Ação para Prevenção e Controle do Desmatamento na Amazônia Legal. Revista do Instituto de Estudos Brasileiros, Brasil, n. 66, p. I08-I29, abr. 2017.

DOI: http://dx.doi.org/Io.II606/issn.23I6-90IX.voi66pIo8-I29

I Universidade de São Paulo (USP, São Paulo, SP, Brasil).

2 Universidade de São Paulo (USP, São Paulo, SP, Brasil). 
Quando se buscam conceitos que elucidem o que são políticas públicas, diversos autores fazem contribuições relevantes, tais como as reunidas por Souza:

Mead (I995) a define [a política pública] como um campo dentro do estudo da política que analisa o governo à luz de grandes questões públicas, e Lynn (I980), como um conjunto de ações do governo que irão produzir efeitos específicos. Peters (I986) segue o mesmo veio: política pública é a soma das atividades dos governos, que agem diretamente ou através de delegação, e que influenciam a vida dos cidadãos. Dye (I984) sintetiza a definição de política pública como "o que o governo escolhe fazer ou não fazer”. Laswell (I958) [define que] decisões e análises sobre política pública implicam responder às seguintes questões: quem ganha o que, por que e que diferença faz. Outras definições enfatizam o papel da política pública na solução de problemas³.

Entende-se, então, que o processo de formulação de uma política pública tem início a partir da detecção de um elemento sobre o qual o governo deve agir. Deve-se definir como tal elemento se configura como um problema a ser solucionado, o porquê da importância de serem dadas soluções a tal problema e quais os resultados esperados ocasionados pelas soluções a serem adotadas. Analisando o modelo de multiple streams proposto por Kingdon em Agendas, alternatives, and public policies, para entendimento a respeito do processo de formação de agendas governamentais, Capella afirma que:

[...] para o modelo de Kingdon, a mudança da agenda é o resultado da convergência entre três fluxos: problemas (problems); soluções ou alternativas (policies); e política (politics).

No primeiro fluxo, o modelo busca analisar de que forma as questões são reconhecidas como problemas e por que determinados problemas passam a ocupar a agenda governamental.

3 SOUZA, Celina. Políticas públicas: uma revisão da literatura. Sociologias, v. 8, n. I6, p. 20-45, 2006. p. 24-25 
Para entender o processo de seleção, Kingdon estabelece uma importante diferenciação entre problemas e questões (conditions). Uma questão, para o autor, é uma situação social percebida, mas que não desperta necessariamente uma ação em contrapartida. Esse tipo de questão configura-se como problema apenas quando os formuladores de políticas acreditam que devem fazer algo a respeito [...]. No segundo fluxo - policy stream - temos um conjunto de alternativas e soluções (policy alternatives) disponíveis para os problemas [...]. As comunidades geradoras de alternativas (policy communities) são compostas por especialistas - pesquisadores, assessores parlamentares, acadêmicos, funcionários públicos, analistas pertencentes a grupos de interesses, entre outros - que compartilham uma preocupação em relação a uma área (policy area) [...]. Finalmente, o terceiro fluxo é composto pela dimensão da política "propriamente dita" (politics stream) [...].

Em determinadas circunstâncias, estes três fluxos - problemas, soluções e dinâmica política - são reunidos, gerando uma oportunidade de mudança na agenda. Nesse momento, um problema é reconhecido, uma solução está disponível e as condições políticas tornam o momento propício para a mudança, permitindo a convergência entre os três fluxos e possibilitando que questões ascendam à agenda [...]. De acordo com o autor, uma oportunidade para a mudança surge quando um novo problema consegue atrair a atenção do governo (por meio de indicadores, eventos ou feedback), ou quando mudanças são introduzidas na dinâmica política [...]. Assim, ao tomarem consciência de um problema, os formuladores de políticas acionam a policy stream em busca de alternativas que apontem soluções para o problema percebido ${ }^{4}$.

No Brasil, as mudanças de uso do solo na Amazônia Legal constituem um exemplo importante de ascensão de uma questão à agenda política. A Floresta Amazônica é caracterizada por uma enorme diversidade de ambientes, com mais de 600 tipos diferentes de hábitats terrestres e de água doce ${ }^{5}$, que abriga rica biodiversidade: cerca de 45 mil espécies de plantas e vertebrados conhecidos ${ }^{6}$, o que corresponde aproximadamente a $1 / 4$ das espécies terrestres globais 7 . A evaporação e a condensação de vapor de água na Floresta Amazônica são motores da circulação atmosférica global, tendo efeitos nas precipitações ao longo da América do Sul e outras regiões ${ }^{8}$. Aproximadamente oito trilhões de toneladas de água evaporam anualmente,

4 CAPELLA, Ana Cláudia. Perspectivas teóricas sobre o processo de formulação de políticas públicas. Revista Brasileira de Informação Bibliográfica em Ciências Sociais - BIB, v. 6I, p. 25-52, 2006. p. 26-30

5 BRASIL. Ministério do Meio Ambiente - MMA \& Serviço Florestal Brasileiro - SFB. Florestas do Brasil em resumo (Relatório). Dados de 2007-20I2. Brasília, DF: MMA e SFB, 20I3, p. 50

6 Ibidem.

7 MALHI, Yadvinder et al. Climate change, deforestation, and the fate of the Amazon. Science, v. 3I9, n. 5.860, p. I69-I72, 2008.

8 Ibidem. 
influenciando o fluxo de calor e a circulação atmosférica global9. Estima-se que na Floresta Amazônica sejam realizados $15 \%$ da fotossíntese terrestre global ${ }^{\text {to }}$ e que a biomassa ali encontrada contenha Ioo bilhões de toneladas de carbono ${ }^{\text {II }}$. Tais fatos tornam evidente que a manutenção da integridade biótica dos serviços ecossistêmicos amazônicos é fundamental.

Em junho de 2003, o Instituto Nacional de Pesquisas Espaciais (Inpe) divulgou dados relativos ao desmatamento na Amazônia Legal no período de 3I de julho de $200 I$ a I ${ }^{\circ}$ agosto de 2002, indicando um crescimento acelerado do desmatamento em torno de $40 \%$ em relação ao ano anterior ${ }^{12}$. Crescentes taxas de desmatamento reconhecidamente ocasionam o desequilíbrio dos serviços ecossistêmicos florestais, acarretando graves impactos ambientais e socioeconômicos. Perante a ameaça de continuidade da tendência de aumentos expressivos nas taxas de desmatamento na Amazônia Legal, a questão das mudanças de uso do solo na região foi reconhecida como um problema para o qual o governo deveria apresentar soluções.

Perante tal desafio, foi assinado o Decreto Presidencial de 3 de julho de 2003, que estabeleceu um Grupo Permanente de Trabalho Interministerial com a finalidade de propor medidas e coordenar ações que visavam à redução dos índices de desmatamento na Amazônia Legal. Foi conduzida, então, uma avaliação cuidadosa das causas do problema, como base para o planejamento de um conjunto de ações integradas do poder público a serem implementadas com a participação ativa da sociedade brasileira. Surgiu, assim, o Plano de Ação para Prevenção e Controle do Desmatamento na Amazônia Legal (PPCDAm), que se constituiu em uma iniciativa estratégica do governo brasileiro e se inseriu plenamente nas diretrizes e prioridades do Plano de Desenvolvimento Sustentável da Amazônia (PAS) ${ }^{\mathrm{r}}$. Assim, o problema do desmatamento na Amazônia passou a integrar o mais alto nível da agenda política do governo federal, envolvendo um grande número de ministérios ${ }^{\mathrm{I}}$.

O presente estudo teve como objetivo analisar a evolução da implementação do PPCDAm. Para tal finalidade, foi realizada análise de: publicações científicas que caracterizam o problema identificado; documentos que compreendem a Fase I (2004-2008), a Fase II (2009-20II) e a Fase III (20I2-20I5) do PPCDAm; avaliações

9 IPCC - Intergovernmental Panel on Climate Change. Summary for policymakers. In: METZ, B.et al. (Ed.). Climate Change 2007 Mitigation. Contribution of Working Group III to the Fourth Assessment Report of the Intergovernmental Panel on Climate Change. Cambridge: Cambridge University Press, United Kingdom and New York, NY, USA, 2007.

Io FIELD, Christopher et al. Primary production of the biosphere: integrating terrestrial and oceanic components. Science, v. 28I, n. 237, I998.

II MALHI, Yadvinder et al., op. cit.

I2 PPCDAm. Plano de Ação para Prevenção e Controle do Desmatamento da Amazônia Legal. Fase I. Brasília,

DF: Casa Civil, 2004.

I3 Ibidem.

I4 PPCDAm. Plano de Ação para Prevenção e Controle do Desmatamento da Amazônia Legal. Fase III. Brasília, DF: Casa Civil, 2012. 
realizadas sobre a Fase I ${ }^{\mathrm{I}}$ e sobre a Fase II do PPCDAm ${ }^{\mathrm{I}}$ (realizada por Ipea; GIZ; Cepal, 20II), encomendadas pelo Ministério do Meio Ambiente (MMA); e publicações científicas que abordam os impactos do PPCDAm na redução do desmatamento. Foram comparadas as diretrizes estratégicas e planos operacionais em cada fase do PPCDAm, observando-se em que medida as avaliações independentes contribuíram para as revisões e a formulação das fases seguintes do Plano. Os impactos do PPCDAm, elencados por diversos cientistas, foram comparados a fim de que se chegasse a um denominador comum, de forma a indicar que eixos de ação contribuíram de forma mais efetiva para a redução do desmatamento.

\section{Caracterização do problema: desmatamento na Amazônia Legal}

As mudanças de uso do solo na Amazônia Legal são altamente heterogêneas, tanto espacial quanto temporalmente. A paisagem atual da região amazônica deriva das diferentes fases de desenvolvimento ocorridas ao longo dos últimos 50 anos. A contribuição das atividades econômicas desenvolvidas na Amazônia Legal para o desmatamento teve início na fase do regime militar (I964 a I985), quando programas específicos de desenvolvimento e ocupação da área estimularam o crescimento populacional e econômico da região. Nesse período, as medidas governamentais não buscavam harmonizar as dimensões sociais, ambientais, políticas e econômicas de desenvolvimento ${ }^{\mathrm{I}}$.

Durante o final dos anos I980 e nos anos I990, projetos de colonização em larga escala, concessão de créditos e investimentos realizados na região levaram à perda de aproximadamente 18 milhões de hectares de área florestada ${ }^{18}$. Laurance et al. ${ }^{19}$ enfatizam o papel crítico da construção de estradas e rodovias e do processo desordenado de ocupação de solo - grilagem de terras - no desmatamento em tal

I5 ABDALA, Guilherme. Plano de Ação para Prevenção e Controle do Desmatamento na Amazônia Legal (PPCDAm) Documento de avaliação 2004-2007. Brasília, DF: Ministério do Meio Ambiente, 2008.

I6 IPEA; GIZ; CEPAL. Avaliação do Plano de Ação para Prevenção e Controle do Desmatamento na Amazônia Legal: PPCDAm 2007-20Io. Brasília: Ipea; GIZ; Cepal, 20II. Disponível em: <http://repositorio.cepal.org/bitstream/ handle/II362/3046/S33375A94520II_pt.pdf;jsessionid=489F2CI4D46I7C7B89I4ABEF88C6CooI?sequence=I > Acesso em: Io maio 2016.

I7 PRATES, Rodolfo Coelho. O desmatamento desigual da Amazônia brasileira: sua evolução, suas causas e consequências sobre o bem-estar. Tese (Doutorado em Ciências). Escola Superior de Agricultura "Luiz de Queiroz”, Universidade de São Paulo, Piracicaba, 2008.

I8 ALVES, Diogenes et al. The changing rates and patterns of deforestation and land use in Brazilian Amazonia. In: KELLER, M. et al. (Ed.). Amazonia and global change. Washington, DC: AGU, 2009. p. II-23. (Geophysics Monograph Series, I86); PRODES. Programa de Monitoramento da Floresta Amazônica Brasileira por Satélite. Ministério do Meio Ambiente - MMA, Instituto Nacional de Pesquisas Espaciais - Inpe, do Ministério da Ciência e Tecnologia. Disponível em: 〈http://www.obt.inpe.br/prodes >. Acesso em: I9 dez. 20I4.

I9 LAURANCE, William et al. Environment: the future of the Brazilian Amazon. Science, v. 29I, n. 5503, p. 438-439, $200 I$. 
período. As correlações entre o desmatamento e o avanço do setor agropecuário tornaram-se mais altas nesse período, especialmente a partir de I994, devido à reforma monetária e ao alcance de estabilidade macroeconômica brasileira, bem como à inserção do país nos mercados globais como importante fornecedor de soja e carne ${ }^{20}$.

Em 200I, uma área de aproximadamente $837.000 \mathrm{~km}^{2}$ da Floresta Amazônica original havia sido devastada no Brasil ${ }^{21}$. A devastação se concentrou no Arco do Desmatamento, nas margens leste e sudeste da Amazônia Legal. No período que compreende os anos de I998-2006, as taxas de desmatamento em tal região foram, em média, de I8.Ioo $\mathrm{km}^{2} \mathrm{ano}^{-\mathrm{I}}$, tendo ocorrido no ano de 2004 o desmatamento de aproximadamente $27.400 \mathrm{~km}^{2}$ das áreas florestadas ${ }^{22}$. Nesse período, as mudanças de uso do solo e consequentes elevadas taxas de desmatamento observadas deveram-se a: I) demandas internacionais crescentes por commodities agroindustriais e escassez de terras apropriadas à expansão agrícola nos EUA, Europa Ocidental, China e outros países ${ }^{23}$; 2) expansão do plantio de cana-de-açúcar para a produção de etanol no estado de São Paulo, o que provocou o deslocamento da produção de soja e rebanhos bovinos $^{24}$; 3) fragilidade das políticas ambientais brasileiras ${ }^{25}$.

De I990 a 2006, o rebanho bovino cresceu a uma taxa média de $6,74 \%$ ao ano na Amazônia Legal, enquanto nas outras regiões do Brasil o crescimento médio do rebanho foi de $0,57 \%$ ao ano ${ }^{26}$. Com essas taxas, segundo os dados da Pesquisa Pecuária Municipal do IBGE, o rebanho cresceu de 26 milhões de cabeças em I990 para 73,7 milhões em 2006, o que representa um aumento de mais de I80\% em I6 $\operatorname{anos}^{27}$. O crescimento acompanhou o aumento da demanda interna e externa de carne bovina.

O cultivo da soja, em particular, representou um acréscimo considerável às atividades agrícolas na Amazônia Legal. A modernização da produção da soja permitiu o extremo avanço da agricultura mecanizada, com a introdução de sistemas de produção altamente capitalizados ${ }^{28}$. $\mathrm{O}$ boom da soja na região, de modo geral,

20 ARIMA, Eugênio et al. Public policies can reduce tropical deforestation: lessons and challenges from Brazil. Land Use Policy, v. 4I, p. 465-473, 2014.

2I SOARES-FILHO, Britaldo et al. Modelling conservation in the Amazon basin. Nature, v. 440, p. 520-523, 2006.

22 MALHI, Yadvinder et al., op. cit.

23 NEPSTAD, Daniel; STICKLER, Claudia; ALMEIDA, Oriana. Globalization of the Amazon soy and beef industries: opportunities for conservation. Conservation Biology, v. 20, n. 6, p. 1595-I603, 2006.

24 NEPSTAD, Daniel et al. Interactions among Amazon land use, forests and climate: prospects for a near-term forest tipping point. Philosophical Transactions of the Royal Society B: Biological Sciences, v. 363, n. I498, p. I737-I746, 2008.

25 Ibidem.

26 RIVERO, Sérgio et al. Pecuária e desmatamento: uma análise das principais causas diretas do desmatamento na Amazônia. Nova Economia, v. I9, n. I, p. 4I-66, 2009.

27 Ibidem.

28 ALVES, Diogenes et al., op. cit. 
representa parte importante do crescimento econômico nacional, com a expansão da produção anual de 20 milhões de toneladas em I990 para 50 milhões em $2004^{29}$.

Atualmente, as atividades pecuárias, a produção de soja, os investimentos em infraestrutura e a grilagem de terras ainda constituem os principais vetores diretos de desmatamento na Amazônia Legal. É importante também ressaltar o papel da indústria madeireira nos processos de desmatamento. A exploração da madeira na Amazônia Legal é um vetor de degradação da floresta e é, em sua maior parte, realizada ilegalmente, exercendo forte pressão sobre unidades de conservação, territórios indígenas e mesmo propriedades particulares. Embora o corte seletivo para extração madeireira não acarrete uma mudança imediata de uso do solo, essa prática geralmente pode levar ao desmatamento ${ }^{30}$. De I999 a 2003, a área onde houve corte seletivo foi similar em magnitude à área desmatada na bacia amazônica ${ }^{31}$. Setzer et al..$^{22}$ demonstraram que um desmatamento ilegal de 520 ha, detectado no início de 20I2, em Feliz Natal (Mato Grosso - MT), foi resultado de processos de degradação e queimadas ilegais que tiveram início no ano de 2004, destacando que a área estudada sofreu maior impacto por queimadas nos anos (secos) de 2007 e 20Io. O estudo evidenciou que os processos de desmatamento e seus impactos ambientais se dão de forma gradativa, e não devem ser "considerados como representativos apenas do ano em que o desmate final foi constatado" 33 .

Conforme citado anteriormente, os processos de desmatamento acarretam diversos impactos ambientais e socioeconômicos, tais como: fragmentação florestal; redução da evapotranspiração; emissões de gases de efeito estufa; redução da biodiversidade; alteração de microclimas; mortalidade de árvores; aumento do risco de incêndios florestais; aumento da concentração de aerossóis; decréscimo do escoamento regional; perda de oportunidades para o uso sustentável da floresta, incluindo a produção de mercadorias tradicionais, tanto por manejo florestal para madeira, como por extração de produtos não madeireiros; maior incidência de doenças tropicais; exclusão social; marginalização de povos indígenas e comunidades tradicionais; perda do conhecimento tradicional; concentração fundiária; e conflitos sociais ${ }^{34}$.

29 Ibidem.

30 DAVIDSON, Eric et al. The Amazon basin in transition. Nature, v. 48I, p. 32I-328, 2012.

3I Ibidem.

32 SETZER, Alberto et al. O caso de um desmate ilegal na Amazônia antecipado pela detecção de fogo e da degradação ambiental. In: SenGeF - SEMINÁRIO DE ATUALIZAÇÃO EM SENSORIAMENTO REMOTO E SISTEMAS DE INFORMAÇÕES GEOGRÁFICAS APLICADOS Ã ENGENHARIA FLORESTAL, IO., Curitiba, 20I2. Disponível em: <http://queimadas.cptec.inpe.br/ rqueimadas/documentos/20I2Io_Setzer_etal_ Desmatellegal_XSengef.pdf $\rangle$. Acesso em: Io maio 2016.

33 Ibidem.

34 DAVIDSON, Eric et al., op. cit.; FEARNSIDE, Philip. Desmatamento na Amazônia: dinâmica, impactos e controle. Acta Amazonica, v. 36, n. 3, p. 395-400, 2006; HECHT, Susanna. From eco-catastrophe to zero deforestation? Interdisciplinarities, politics, environmentalisms and reduced clearing in Amazonia. Envir. Conserv., v. 39, n. oI, p. 4-I9, 20II. 
A enumeração de tais impactos torna evidente a necessidade de manutenção de baixas taxas de desmatamento. Frente à ameaça de continuidade da tendência de aumentos significativos da taxa de desmatamento anual na Amazônia Legal, o governo estabeleceu a implementação do PPCDAm. A seguir, tal plano será detalhado, em suas três fases.

\section{PPCDAM FASE I (2004-2008)}

Sob a coordenação da Casa Civil da Presidência da República, o Grupo Permanente de Trabalho Interministerial foi composto com a participação dos títulos dos seguintes órgãos: Ministério da Agricultura, Pecuária e Abastecimento (Mapa); Ministério da Ciência e Tecnologia (MCT); Ministério da Defesa (MD); Ministério do Desenvolvimento Agrário (MDA); Ministério do Desenvolvimento, Indústria e Comércio Exterior (MDIC); Ministério da Integração Nacional (MI); Ministério da Justiça (MJ); Ministério do Meio Ambiente (MMA); Ministério das Minas e Energia (MME); Ministério dos Transportes (MT); e Ministério do Trabalho e Emprego (MTE). A partir do decreto assinado em I5 de março de 2004, passaram a integrar o grupo o Ministério do Planejamento, Orçamento e Gestão e o Ministério das Relações Exteriores ${ }^{35}$. A reunião de vários ministros de Estado para coordenar e integrar a intervenção do governo nesse tema consistiu-se em uma forma até então pouco usual e desafiadora para a estrutura rígida da administração pública ${ }^{36}$.

Com a criação do GT Interministerial sobre o Desmatamento na Amazônia, foi tomado como primeiro passo o estabelecimento de quatro subgrupos de trabalho para a elaboração de propostas estratégicas, detalhados na tabela I.

35 PPCDAm, op. cit., 2004.

36 PPCDAm, op. cit., 2009. 


\begin{tabular}{|l|l|l|}
\hline \multicolumn{1}{|c|}{ Subgrupo } & \multicolumn{1}{|c|}{ Membros } & \multicolumn{1}{c|}{ Área de atuação } \\
\hline $\begin{array}{l}\text { O r d e n a m e n t o } \\
\text { Fundiário e Territorial }\end{array}$ & $\begin{array}{l}\text { SDS/MMA (coord.), SCA/MMA, } \\
\text { MDA, Mapa, MI, MDIC, MD, } \\
\text { MJ/Funai }\end{array}$ & $\begin{array}{l}\text { Instrumentos de ordenamento } \\
\text { territorial com enfoque para } \\
\text { política fundiária, unidades } \\
\text { de conservação e estratégias } \\
\text { de de senvolvi mento local } \\
\text { sustentável. }\end{array}$ \\
\hline $\begin{array}{l}\text { Mon i tor a me nto e } \\
\text { Controle }\end{array}$ & $\begin{array}{l}\text { MMA, MCT, MD, MJ, MTE, } \\
\text { Sipam/CasaCivil }\end{array}$ & $\begin{array}{l}\text { Instrumentos de monitoramento, } \\
\text { licenciamento e fiscalização de } \\
\text { desmatamento, queimadas e } \\
\text { exploração madeireira. }\end{array}$ \\
\hline $\begin{array}{l}\text { Fomento a Atividades } \\
\text { Sustentáveis }\end{array}$ & $\begin{array}{l}\text { SBF/MMA (coord.), SCA/MMA, } \\
\text { MDIC, Mapa, MDA, MCT, MI, } \\
\text { MTE, MF (convidado) }\end{array}$ & $\begin{array}{l}\text { Assistência técnica e extensão } \\
\text { rural. } \\
\text { Pesquisa científica e tecnológica. }\end{array}$ \\
\hline $\begin{array}{l}\text { Infraestrutura } \\
\text { SCA/MMA (coord.), MT, MME, } \\
\text { Mapa, MI, MDIC }\end{array}$ & $\begin{array}{l}\text { Políticas de infraestrutura, } \\
\text { com enfoque para os setores de } \\
\text { transporte e energia. }\end{array}$ \\
\hline
\end{tabular}

Tabela I - Subgrupos de trabalho e áreas de atuação. Fonte: adaptado de PPCDAm, 2004

Nessa primeira fase do plano, as medidas de combate ao desmatamento tiveram as seguintes diretrizes: I) valorização da floresta para fins de conservação e uso sustentável; 2) recuperação de áreas degradadas como forma de aumentar a produtividade e diminuir as pressões sobre as florestas remanescentes; 3 ) ordenamento fundiário e territorial priorizando o combate à grilagem de terras públicas, a criação de unidades de conservação e a homologação de terras indígenas; 4) aprimoramento dos instrumentos de monitoramento, licenciamento e fiscalização do desmatamento; 5) fomento às atividades de uso sustentável dos recursos florestais e/ou uso intensivo de áreas agrícolas; 6) gestão descentralizada e compartilhada de políticas públicas entre União, estados e municípios; e 7) participação ativa dos diferentes setores interessados da sociedade amazônica na gestão das políticas relacionadas à prevenção e controle do desmatamento ${ }^{37}$.

O Plano Operacional foi idealizado no âmbito de cada subgrupo de trabalho, descrito conforme segue: 
- Monitoramento e Controle. A grande inovação no monitoramento e controle sobre o desmatamento foi a implantação do sistema integrado de "alerta", o qual permitiu em tempo quase real a detecção, através de imagens de satélite, do desmatamento: o Sistema Deter (Detecção do Desmatamento em Tempo Real). Além disso, o governo federal, também de forma conjunta com os governos estaduais, intensificou as investigações sobre crimes ambientais de maneira integrada, usando as informações disponíveis nos órgãos vinculados. Isso permitiu sobrepor informações trabalhistas, ambientais, fiscais, tributárias e fundiárias para rastrear atividades atreladas ao desmatamento ilegal ${ }^{3}$.

- Ordenamento Fundiário e Territorial. O governo federal priorizou as ações de ordenamento fundiário e territorial ao longo do Arco do Desmatamento, com destaque para a área de influência da BR I63 (rodovia Santarém-Cuiabá). As ações emergenciais incluíram o combate à grilagem de terras públicas, a criação de novas unidades de conservação (tanto de uso sustentável como de proteção integral) e a demarcação e homologação de terras indígenas. De maneira complementar, o governo federal, em parceria com os governos estaduais (Pará, Mato Grosso, Rondônia e Acre) e a sociedade civil, atuou conjuntamente para realizar o zoneamento ecológico-econômico (ZEE) ao longo do Arco do Desmatamento e da área de influência da BR I63. Em áreas já desmatadas e naquelas indicadas através do ZEE para uso agrícola, o governo promoveu a expansão de atividades agrícolas em bases sustentáveis ${ }^{39}$.

- Fomento a Atividades Sustentáveis. Foi essencial alterar substancialmente os instrumentos de fomento para apoiar o uso sustentável dos recursos naturais na Amazônia. No âmbito dos fundos constitucionais, foram definidas novas diretrizes e critérios para o uso sustentável dos recursos naturais. Além disso, o Protocolo Verde foi aperfeiçoado para implementação pelos bancos públicos e privados. $\mathrm{O}$ governo federal, em parceria com os governos estaduais, a sociedade civil e o setor empresarial, promoveu também a intensificação de um programa de capacitação de mão de obra com ênfase no manejo florestal e agricultura intensiva, essa última em áreas já desmatadas ${ }^{4}$.

- Infraestrutura. Nas últimas décadas, os grandes investimentos em infraestrutura -especialmente as rodovias - têm sido uma das principais causas do desmatamento na Amazônia Legal. O governo federal e os governos estaduais promoveram, então, a coordenação do planejamento estratégico de obras de infraestrutura e medidas preventivas, mitigadoras e compensatórias a serem executadas antes da realização das obras ${ }^{4}$.

Porém, com o objetivo de que a atuação do PPCDAm fosse restringida para o campo diretamente vinculado ao problema central que propõe solucionar, houve a migração do componente "infraestrutura ambientalmente sustentável” para o PAS, já em

38 Ibidem.

39 Ibidem.

40 Ibidem.

4I Ibidem. 
$2004^{42}$. Dessa forma, os subgrupos se estabeleceram como: Ordenamento Fundiário e Territorial; Monitoramento e Controle; e Fomento a Atividades Sustentáveis.

Após quatro anos de sua implementação, novos fatos deixaram clara a necessidade de uma avaliação pormenorizada do PPCDAm, para indicar acertos e correções a serem feitos nas políticas de combate ao desmatamento. Para tal, no final de 2007, o Ministério do Meio Ambiente contratou uma consultoria independente para a realização de uma avaliação do PPCDAm Fase I, que será detalhada a seguir.

\section{Avaliação do PPCDAm Fase I}

Tal avaliação foi organizada e realizada pelo engenheiro-agrônomo Guilherme C. Abdala e sua equipe no ano de 2008. O foco do trabalho foi analisar a evolução, as dificuldades e as lições aprendidas após quatro anos do lançamento do PPCDAm, além de apontar questões a serem consideradas durante o processo de revisão para a segunda fase do Plano ${ }^{43}$.

Para avaliação e revisão do PPCDAm, foram desenvolvidas as seguintes atividades: levantamento e análises documentais; realização de entrevistas nos ministérios e órgãos envolvidos com o Plano; realização de entrevistas com governos estaduais (Oemas); e realização de entrevistas com representantes de ONGs e de movimentos sociais 4 .

Em relação à formulação do PPCDAm, a avaliação conduzida por Abdala ${ }^{45}$ apontou: a falta de clareza e/ou detalhamento das relações que deveriam ser articuladas, ou que remetessem a um processo de articulação sistematizado, com grupos de interesse diversos - em especial os poderes públicos estaduais e municipais, representantes da iniciativa privada e organizações da sociedade civil; a projeção de várias ações de forma abrangente e generalizada, sem foco e/ou objetividade específicas, ou seja, sem conhecimento claro de particularidades ou peculiaridades sub-regionais e microrregionais; e a dificuldade da coordenação central do PPCDAm em prever os constrangimentos que se sucederam relacionados ao esforço multissetorial, principalmente no quesito transversalidade.

A respeito do desempenho dos subgrupos durante os quatro anos de implementação do Plano, Abdala ${ }^{46}$ afirmou que estes apresentaram desempenhos diferenciados, assim verificados: Subgrupo I - Ordenamento Fundiário: desempenho intermediário; Subgrupo II - Monitoramento e Controle: melhor desempenho; Subgrupo III - Fomento a Atividades Sustentáveis: desempenho baixo. No PPCDAm, funcionaram bem as ações que já eram conhecidas, que faziam parte do dia a dia das instituições responsáveis, as quais sabiam como e onde atuar. O desequilíbrio no desempenho dos subgrupos, durante os quatro anos decorridos, foi um dos

\footnotetext{
42 PPCDAm, op. cit., 2009.

43 ABDALA, Guilherme, op. cit.

44 Ibidem.

45 Ibidem.

46 Ibidem.
} 
fatores determinantes que reduziram a sustentabilidade e a efetividade das ações do PPCDAm.

As diretrizes para revisão do Plano para sua segunda fase foram sugeridas por Abdala ${ }^{47}$ : I) um desenho mais claro, relacionando o objetivo superior (diminuição da taxa de desmatamento) e respectivas interações de causalidade, diretas ou indiretas, com ações e atividades propostas; 2) a especificação clara de dimensões quantitativas, temporais e espaciais, ou seja, das metas relacionadas aos objetivos de projetos e, também, de objetivo(s) superior(es); 3) uma padronização hierárquica do conjunto de subgrupos, ações e atividades, assim como do respectivo conjunto de indicadores; 4) a estruturação, para cada componente, das ações estratégicas e respectivos graus de prioridade, conforme teia de causalidade do desmatamento.

Abdala ${ }^{48}$ sugeriu, ainda, para uma conformação adequada do processo de planejamento, gestão e monitoramento do PPCDAm, a adoção da metodologia do Marco Lógico, devendo ser formulada em conjunto com o Ministério do Planejamento. O Marco Lógico consiste em uma estrutura de implicações lógicas de causa-efeito, com relação a uma situação-problema, e de meios-fins em relação à intervenção proposta para mudar a situação-problema. Consiste, portanto, em um conjunto de conceitos inter-relacionados, que definem as causas de uma intervenção (projeto), bem como o que deve ser feito (estratégia) para alcançar o resultado desejado 49 .

Considerando o interesse de se estruturar o PPCDAm de forma a potencializar seu processo de monitoramento constante, Abdala $^{50}$ recomendou que, por meio do Marco Lógico, deveriam ser estabelecidos: I) uma Árvore de Problemas, consistindo em um sistema hierarquizado de relações causa-efeito de um problema original; 2) uma Árvore de Objetivos ou Árvore de Soluções, construída a partir dessa cadeia de causalidade para propor um conjunto de relações meios-fins como alternativas de solução para o problema original; 3) uma Matriz de Decisão, a partir da qual se passa ao planejamento da intervenção propriamente dita, com base em estratégias disponíveis baseadas em potenciais e limitações correntes.

Do ponto de vista temático, Abdala afirmou que os seguintes aspectos deveriam ser ressaltados para a revisão do Plano: I) sustentabilidade política do PPCDAm no médio e longo prazos; 2) controle social e transparência (legitimação do PPCDAm); 3) descentralização, governança ambiental e protagonismo dos governos estaduais; 4) articulação com estratégias para redução das emissões de $\mathrm{CO}_{2}$; 5) estratégia de responsabilização compartilhada pelo desmatamento; 6) foco em municípios prioritários.

Como proposição inicial de revisão do PPCDAm, sugeriu-se a inclusão de um novo subgrupo, que deveria estar relacionado a ações que necessitariam receber novo grau

\footnotetext{
47 Ibidem.

48 Ibidem.

49 Ibidem.

50 Ibidem.
} 
de importância na segunda fase, além de articulação no âmbito horizontal e vertical. Abdala ${ }^{51}$ sugeriu que esse novo subgrupo fosse denominado "Governança Ambiental".

A partir da avaliação dos pontos levantados e sugestões oferecidas por Abdala e sua equipe técnica, o modelo lógico do PPCDAm foi apresentado e discutido com os secretários e representantes das secretarias de Meio Ambiente dos Estados da Amazônia Legal e representantes de organizações não governamentais (ONGs). As sugestões, críticas e demandas foram avaliadas e foi elaborada a segunda fase do Plano, detalhada a seguir.

\section{PPCDAM FASE II (2009-20II)}

Após os primeiros anos de implementação da primeira fase do PPCDAm, questões relacionadas às mudanças de uso do solo e desmatamento na Amazônia Legal apontaram sinais de modificações. Em relação à dinâmica do desmatamento em si, os grandes polígonos de desmatamento proeminentes nos anos anteriores passaram a diminuir sua participação no total do desmatamento, o que levou a um aumento relativo dos pequenos polígonos. Também, em 2007, os alertas mensais emitidos pelo Sistema Deter começaram a apontar uma retomada do aumento nas taxas do desmatamento. Os meses de chuva, como novembro e dezembro, que geralmente contam com pouco desmatamento, registraram até $1.000 \mathrm{~km}^{2}$ de desmatamento ${ }^{52}$. Em relação à gestão do problema, um ponto importante foi a descentralização da gestão florestal para os estados, ocorrida em 2006, por meio da Lei de Gestão de Florestas Públicas. Destaca-se, também, o lançamento do Plano Nacional sobre Mudança do Clima (PNMC), trazendo metas quadrienais de redução do desmatamento na Amazônia.

A segunda fase do PPCDAm, então, refletiu tais mudanças. As diretrizes incorporadas foram: I) valorização da floresta para fins de conservação da biodiversidade, manejo florestal de produtos madeireiros e não madeireiros e prestação de serviços ambientais; 2) incentivos para a melhor utilização de áreas já desmatadas, contemplando inovação tecnológica e sistemas sustentáveis de produção; 3) apoio a processos de certificação e valorização dos produtos da biodiversidade e de agregação de valor a esses produtos e a produtos oriundos de atividades locais; 4) modelos alternativos de reforma agrária adequados à Amazônia, e criação e consolidação de mais unidades de conservação e terras indígenas; 5) adoção de um estilo de gestão descentralizada e compartilhada de políticas públicas, por meio de parcerias entre União, estados e municípios; 6) estímulo à participação ativa dos diferentes setores da sociedade amazônica interessados na gestão das políticas relacionadas ao controle do desmatamento; 7) incentivo à implementação do Cadastro Ambiental Rural (CAR), instrumento por meio do qual os órgãos ambientais dispõem do georreferenciamento de imóveis rurais, de modo a qualificar o monitoramento remoto e a efetividade das operações de fiscalização em campo, bem como orientar

5 I Ibidem.

52 PPCDAm, op. cit., 2009. 
o processo de regularização ambiental do imóvel rural; e 8) dar visibilidade à realização das ações do Plano por meio da divulgação das informações, envolvendo as assessorias de comunicação dos ministérios e da Presidência da República53.

Outras medidas significativas associadas à segunda fase do Plano são descritas na tabela 2.

\begin{tabular}{|c|c|}
\hline Medida & Descrição \\
\hline $\begin{array}{l}\text { Resolução } \\
\text { CMN/Bacen n. } \\
3.545 / 2008- \\
\text { Concessão de } \\
\text { Crédito }\end{array}$ & $\begin{array}{l}\text { O crédito deve ser seguido da adoção de mecanismos de controle por parte } \\
\text { dos órgãos ambientais, como o georreferenciamento dos imóveis e das áreas } \\
\text { de conservação, a fim de evitar que recursos públicos sejam vinculados ao } \\
\text { desmatamento ilegal. }\end{array}$ \\
\hline $\begin{array}{l}\text { Decreto } 7.008 \text { - } \\
\text { Operação Arco } \\
\text { Verde }\end{array}$ & $\begin{array}{l}\text { O Decreto } 7.008 \text { instituiu a Operação Arco Verde no âmbito do PPCDAm e } \\
\text { criou o seu Comitê Gestor Nacional. Seu propósito é o de promover modelos } \\
\text { produtivos sustentáveis nos municípios considerados prioritários para o } \\
\text { controle e a redução do desmatamento na Amazônia Legal. }\end{array}$ \\
\hline Planos estaduais & $\begin{array}{l}\text { A necessidade de maior integração entre as açães federais e estaduais levou } \\
\text { o governo federal, por meio do MMA, a executar um projeto de cooperação } \\
\text { técnica com o Programa das Nações Unidas para o Desenvolvimento - PNUD } \\
\text { e com doação do Reino da Noruega, com o objetivo de apoiar a elaboração } \\
\text { de planos de prevenção e controle do desmatamento nos estados do Acre, } \\
\text { Mato Grosso e Pará. Com recursos do Programa Piloto para a Proteção das } \\
\text { Florestas Tropicais do Brasil (PPG7), foi possível também apoiar a elaboração } \\
\text { dos planos estaduais de Tocantins e Rondônia. }\end{array}$ \\
\hline $\begin{array}{l}\text { Formulação de } \\
\text { pactos setoriais }\end{array}$ & $\begin{array}{l}\text { Pacto pela Valorização da Floresta e pelo Fim do Desmatamento na Amazônia } \\
\text { Brasileira; Pacto pela Madeira Legal e Desenvolvimento Sustentável; } \\
\text { Protocolos de Intenções pela Responsabilidade Socioambiental entre MMA, } \\
\text { BNDES, CEF, Basa, BB e BNE e entre MMA e Febraban (Novo Protocolo Verde); } \\
\text { Moratória da Soja; Acordo de Cooperação Técnica entre MMA, Ibama e CEF e } \\
\text { Protocolo de Intenções entre MMA e Fiesp. }\end{array}$ \\
\hline
\end{tabular}

Tabela 2 - Medidas de combate ao desmatamento associadas à implementação da segunda fase do PPCDAm. Fonte: elaborada pela autora a partir de dados presentes em PPCDAm, 2009

Percebe-se que grande parte das sugestões surgidas a partir da avaliação realizada sobre a primeira fase do PPCDAm por Abdala ${ }^{54}$ foram incorporadas na definição das diretrizes estratégicas que compõem a sua segunda fase. Destaca-se que tais diretrizes foram elaboradas a partir da adoção da metodologia do Marco Lógico e que foram definidos um Plano Operativo, que contempla "macroações", e um Plano Operativo, que contempla “ações complementares”, refletindo a necessidade,

53 Ibidem.

54 ABDALA, Guilherme, op. cit. 
apontada por Abdala ${ }^{55}$, de estruturação das ações estratégicas e definição de graus de prioridade. No PPCDAm Fase II encontra-se desenhada a Árvore de Problemas também sugerida, porém esta se apresenta de forma ilegível. Os eixos de atuação/ subgrupos permaneceram sendo: Ordenamento Territorial; Monitoramento e Controle; e Fomento a Atividades Sustentáveis.

Em 20Io, o MMA e a Comissão Executiva do PPCDAm detectaram a necessidade de realização de uma avaliação sobre a segunda fase do Plano. Assim, entre outubro de 2010 e julho de 20II, tal avaliação foi conduzida conjuntamente pelo Instituto de Pesquisa Econômica Aplicada (Ipea), pela Comissão Econômica para a América Latina e o Caribe (Cepal) e pela Cooperação Alemã para o Desenvolvimento, por meio da Deutsche Gesellschaftfür Internationale Zusammenarbeit (GIZ). Tal avaliação será detalhada a seguir.

\section{Avaliação do PPCDAm Fase II}

A avaliação conduzida por membros do Ipea, Cepal e GIZ apresentou, como recomendações referentes a aspectos gerais, os seguintes pontos: I) manter a importância política do PPCDAm e uma coordenação de alto nível no âmbito do governo federal; 2) promover ações que visem a diminuir os entraves burocráticos para a regularização fundiária e ambiental e para a promoção de atividades produtivas sustentáveis, particularmente para as pequenas propriedades; 3) aprimorar a diferenciação regional das estratégias, levando em consideração as zonas previstas no Macrozoneamento da Amazônia Legal, assim como as orientações dos zoneamentos estaduais; 4) manter o foco específico nos municípios que mais desmatam, considerando a inserção regional desses municípios para evitar o efeito de vazamento do desmatamento; 5) estruturação de cadeias produtivas sustentáveis; 6) regularização fundiária (prioridade máxima) ${ }^{56}$.

Em relação a recomendações mais específicas, a tabela 3 traz uma síntese delas.

55 Ibidem.

56 IPEA; GIZ; CEPAL. Avaliação do Plano de Ação para Prevenção e Controle do Desmatamento na Amazônia Legal: PPCDAm 2007-20Io. Brasília: Ipea; GIZ; Cepal, 20II. Disponível em: <http://repositorio.cepal.org/bitstream/ handle/II362/3046/S33375A94520II_pt.pdf;jsessionid=489F2CI4D46I7C7B89I4ABEF88C6CooI?sequence=I > Acesso em: Io maio 2016. 


\begin{tabular}{|c|c|}
\hline Área de atuação & Recomendações \\
\hline Estrutura e planejamento & $\begin{array}{l}\text { Atualização da Árvore de Problemas. } \\
\text { As ações do PPCDAm planejadas a partir dos problemas } \\
\text { identificados na Árvore. } \\
\text { Maior complementaridade e sequenciamento lógico das } \\
\text { ações entre os eixos e dentro de cada um deles. } \\
\text { Conjunto de metas com indicadores de impacto } \\
\text { verificáveis através do sistema de monitoramento para } \\
\text { os três eixos. }\end{array}$ \\
\hline $\begin{array}{l}\text { Planos estaduais de controle } \\
\text { do desmatamento }\end{array}$ & Planejamento conjunto e metas coerentes. \\
\hline Municípios prioritários & $\begin{array}{l}\text { Apoio a pactos locais. } \\
\text { Reformular políticas para saída da lista de municípios } \\
\text { prioritários. } \\
\text { Criar incentivos para saída da lista. Formular políticas } \\
\text { "pós-saída da lista”. }\end{array}$ \\
\hline $\begin{array}{l}\text { Macrozoneamento da Amazônia } \\
\text { Legal }\end{array}$ & $\begin{array}{l}\text { Ordenamento territorial local nos municípios } \\
\text { prioritários. } \\
\text { Realização de ZEEs setoriais específicos para as mais } \\
\text { importantes cadeias produtivas da região. }\end{array}$ \\
\hline $\begin{array}{l}\text { Unidades de conservação e terras } \\
\text { indígenas }\end{array}$ & $\begin{array}{l}\text { Redefinir a estratégia de criação de UCs e TIs. } \\
\text { Focalizar a criação de UCs em hotspots. } \\
\text { Valorização econômica das áreas protegidas. }\end{array}$ \\
\hline Ordenamento fundiário & $\begin{array}{l}\text { Reformulação da estrutura institucional. } \\
\text { Sistema cartorial integrado e acessível. } \\
\text { Integração Programa Terra Legal, CAR e licenciamento. } \\
\text { Destinação de terras públicas e ZEEs. } \\
\text { Regularização ocupacional de assentamentos. }\end{array}$ \\
\hline Monitoramento do desmatamento & $\begin{array}{l}\text { Aperfeiçoamento dos sistemas. } \\
\text { Previsão de futuros desmatamentos. } \\
\text { Disponibilização de mapas. } \\
\text { Integração entre instituições e entes federativos. } \\
\text { Rastreabilidade de cadeias produtivas. } \\
\text { Compartilhamento de informações. }\end{array}$ \\
\hline $\begin{array}{l}\text { Reformulação do eixo fomento a } \\
\text { atividades sustentáveis }\end{array}$ & $\begin{array}{l}\text { Reformulação do eixo de forma a superar sua estrutura } \\
\text { ainda caracterizada pela sobreposição de ações e } \\
\text { atividades que não são articuladas em torno de um } \\
\text { objetivo único. }\end{array}$ \\
\hline Diálogo com o setor privado & $\begin{array}{l}\text { Consolidação e fomento do diálogo com o setor privado } \\
\text { e o maior envolvimento desse e das organizações da } \\
\text { sociedade civil nas atividades que promovam a redução } \\
\text { do desmatamento. }\end{array}$ \\
\hline Manejo florestal sustentável & $\begin{array}{l}\text { Aperfeiçoar o marco legal sobre o uso dos recursos } \\
\text { genéticos e dos conhecimentos tradicionais a ele } \\
\text { associados, para permitir atividades de bioprospecção, } \\
\text { pesquisa e exploração de novos produtos. }\end{array}$ \\
\hline
\end{tabular}

Tabela 3 - Síntese de Recomendações para Revisão do PPCDAm Fase II. Fonte: elaborada pela autora a partir de dados presentes em IPEA; CEPAL; GIZ, 20II 
Com base nas recomendações da avaliação, iniciou-se mais um processo de revisão do PPCDAm, que contou com apoio metodológico da Secretaria de Planejamento e Investimentos Estratégicos (SPI), do Ministério do Planejamento, Orçamento e Gestão ${ }^{57}$. Após tal processo, foi formulada a terceira fase do Plano, descrita a seguir.

\section{PPCDAM FASE III (2012-20I5)}

Na primeira e na segunda fases do PPCDAm (de 2004 a 20II), as ações de maior impacto na queda do desmatamento partiram do eixo Monitoramento e Controle, estando bastante associadas ao desenvolvimento do Sistema Deter e ao planejamento integrado da fiscalização. Atualmente, no entanto, o padrão do desmatamento apresenta mudanças, fazendo com que a maior parte dos desmatamentos encontre-se abaixo do limiar de detecção do Deter. A redução na área dos polígonos e a sua dispersão (pulverização) aumentam, consequentemente, o custo da fiscalização, que é limitado tanto por recursos humanos quanto orçamentários. Portanto, a redução dos índices anuais de desmatamento até o ano de 2020 em pelo menos $80 \%$ em relação à média verificada entre os anos de 1996 a 2005, na Amazônia Legal, dependerá também do alcance das políticas públicas aos polígonos inferiores a 25 hectares, através do fortalecimento dos eixos de Ordenamento Fundiário e Territorial e Fomento às Atividades Produtivas Sustentáveis. Nesse contexto, o PPCDAm iniciou sua terceira fase de execução (20I2-20I5) com um desafio ainda maior: promover ações condizentes com a nova dinâmica do desmatamento e dar escala e eficácia ao eixo de Fomento às Atividades Produtivas Sustentáveis ${ }^{5}$.

As diretrizes gerais da nova fase do Plano são: I) adoção de um estilo de gestão descentralizada e compartilhada de políticas públicas, por meio de parcerias entre a União, estados e municípios, contemplando a sua integração com incentivos à prevenção de danos ambientais e ao fomento de sistemas sustentáveis de produção; 2) estímulo à participação ativa dos diferentes setores da sociedade amazônica interessados na gestão das políticas relacionadas ao controle do desmatamento, como meio para aumentar a qualidade de sua implementação, com transparência, controle social e apropriação política; 3) apoiar a implementação dos Planos Estaduais de Prevenção e Controle do Desmatamento na Amazônia Brasileira; 4) ampliar e incentivar os pactos setoriais como forma de firmar o comprometimento de entidades dos setores produtivos (soja, madeira, carvão vegetal), visando à preservação e conservação das florestas; e 5) dar visibilidade à realização das ações do PPCDAm.

O novo modelo lógico apontou um conjunto de elementos que foram agrupados por objetivos estratégicos, escolhidos por configurarem as causas primárias, a saber:

57 PPCDAm, op. cit., 2012.

58 Ibidem. 
- Eixo Ordenamento Fundiário e Territorial: promover ordenamento fundiário de terras públicas; implementar os instrumentos de ordenamento territorial visando à conservação da floresta; realizar a gestão da malha fundiária de acordo com as diversas categorias fundiárias ${ }^{59}$.

- Eixo Monitoramento e Controle: tornar mais célere o licenciamento dos Planos de Manejo Florestal e as Concessões Florestais; aumentar a eficácia da fiscalização e do controle do desmatamento; aumentar a presença do Estado na Amazônia Legal; reduzir a impunidade administrativa e criminal relacionada ao desmatamento ilegal; promover a responsabilização ambiental das principais cadeias produtivas relacionadas ao desmatamento ilegal ${ }^{60}$.

- $\quad$ Eixo Fomento às Atividades Produtivas Sustentáveis: promover a viabilidade das cadeias produtivas que constituem alternativas ao desmatamento; fomentar boas práticas agropecuárias, incluindo a substituição do uso do fogo; aumentar a produção e a comercialização de madeira por meio do manejo florestal sustentável; promover adequação ambiental e fomentar atividades produtivas sustentáveis nos assentamentos da reforma agrária e na agricultura familiar; gerar capacitação, tecnologia e informação sobre a Amazônia de modo a subsidiar o desenvolvimento sustentável ${ }^{6 \mathrm{~T}}$.

Para cada eixo de atuação, foram definidas, ainda: diretrizes específicas; área prioritária de atuação; impactos esperados e ações que demandam maior integração com os estados da Amazônia Legal. O eixo de atuação Fomento às Atividades Sustentáveis é aquele que apresenta maior detalhamento na definição de diretrizes e impactos esperados.

Percebe-se que tal estruturação reflete as recomendações do grupo técnico composto por Ipea, Cepal e GIZ em sua avaliação realizada entre 20Io e 20II, conforme mencionado anteriormente. O eixo de Fomento às Atividades Sustentáveis apresentou nova estrutura de planejamento, conforme recomendado pela avaliação. Também é detalhado um novo modelo de governança, que se justifica pela demanda de acompanhamento continuado da execução das ações do Plano. O novo modelo de governança do PPCDAm se divide em três esferas: Executiva, Consultiva e de Transparência. Tal configuração facilita a correção de rumos, a tomada de decisão do Ministério do Meio Ambiente como órgão coordenador e a solução de problemas e conflitos que eventualmente possam surgir entre órgãos federais e mesmo entre esses e os estaduais ${ }^{62}$.

\footnotetext{
59 Ibidem.

60 Ibidem.

6I Ibidem

62 Ibidem.
} 


\section{IMPACTOS DO PPCDAM NA REDUÇÃO DE DESMATAMENTO}

Em 2005, um ano após o primeiro ano de implementação da primeira fase do PPCDAm, a taxa de desmatamento na Amazônia Legal apresentou expressiva redução, e tal tendência foi mantida até o ano de $2013^{63}$. Tal redução foi resultado de uma sinergia de fatores, sendo a implementação do PPCDAm um deles. Os principais efeitos do plano foram a criação e a expansão de áreas protegidas e a implementação do Sistema Deter.

A expansão da rede de Áreas Protegidas na Amazônia Brasileira é citada como uma das principais contribuições das medidas implementadas pelo PPCDAm para a redução das taxas de desmatamento na região. Soares-Filho et al. ${ }^{64}$ estimam que a criação de novas Áreas Protegidas na Amazônia Brasileira a partir de 2002 foi responsável por $37 \%$ do declínio de $13.400 \mathrm{~km}^{2}$ na taxa de desmatamento observado entre os anos de 2004 e 2006, afirmando que não foi encontrada dependência espacial entre regiões em que houve a expansão de tais áreas e as regiões em que as taxas de desmatamento aumentaram.

Apesar de áreas protegidas virem sendo criadas antes da implantação do PPCDAm, este alavancou a expansão delas, instituindo a criação de mais de 50 milhões de hectares em unidades de conservação federais e estaduais, e a homologação de Io milhões de hectares em terras indígenas, majoritariamente localizadas nas áreas sob pressão de desmatamento ${ }^{65}$.

Em relação à implementação do Sistema Deter, Assunção, Gandour e Rocha ${ }^{66}$ afirmam que tal medida foi o principal motor de desaceleração das taxas de desmatamento na Amazônia Brasileira e estimam que as políticas de comando-econtrole baseadas em tal sistema impediram o desmatamento de mais de 59.500 $\mathrm{km}^{2}$ de Floresta Amazônica, entre os anos de 2007 e 20II, devido à ação reforçada dos agentes do Ibama na região, ressaltando que, em tal cenário, não houve o comprometimento da produção agrícola. Rajão e Vurdubakis ${ }^{67}$ afirmam, também, que a introdução do Sistema Deter e as tecnologias associadas a ele trouxeram ganhos expressivos por terem possibilitado um crescimento expressivo no número de multas aplicadas pelo Ibama quando da verificação de ocorrência de desmatamento ilegal.

É importante salientar que o Sistema Deter funciona como polo irradiador de ações, estando presente em vários lugares ao mesmo tempo e tendo sido utilizado para

63 PRODES, op. cit.

64 SOARES-FILHO, Britaldo et al. Role of Brazilian Amazon protected areas in climate change mitigation.

Proceedings of the National Academy of Sciences, v. I07, n. 24, p. I082I-I0826, 2010.

65 PPCDAm, op. cit., 2012.

66 ASSUNÇÃO, Juliano; GANDOUR, Clarissa; ROCHA, Romero. DETERring deforestation in the Brazilian Amazon: environmental monitoring and law enforcement. Núcleo de Avaliação de Políticas Climáticas da Pontifícia Universidade Católica do Rio de Janeiro (NAPC/PUC-Rio) \& Climate Policy Initiative Rio de Janeiro (CPI Rio), 2013.

67 RAJÃO, Raoni; VURDUBAKIS, Theo. On the pragmatics of inscription: detecting deforestation in the Brazilian Amazon. Theory, Culture er Society, v. 30, n. 4, p. I5I-I77, 2013. 
diversas finalidades. Foi a partir dos dados gerados pelo Sistema que foi detectada, no segundo semestre de 2007, a necessidade da criação da lista de municípios prioritários onde exigências adicionais que visam ao combate ao desmatamento devem ser aplicadas. Além disso, a implementação do sistema ocasionou a mudança comportamental por parte do agente provocador de desmatamento, que passou a ter ciência de que está sendo monitorado e passou a desmatar áreas inferiores a 25 ha, que não são detectadas pelas imagens dos satélites utilizados no Sistema ${ }^{68}$. A percepção do setor empresarial (indústrias pecuárias e agrícolas) passou a ser uma em que os riscos associados ao desmatamento (considerando-se multas agora aplicadas de forma mais efetiva e o risco de embargo de propriedades) de grandes áreas se tornaram mais caros do que os custos para sua realização.

\section{Conclusão}

A redução das taxas de desmatamento na região amazônica constatada entre os anos de 2005 e $2013^{69}$ apresenta inegável relação com a implementação e aperfeiçoamento do Plano de Combate e Controle do Desmatamento na Amazônia Legal. Porém, o desmatamento na Amazônia Legal apresenta um grau de complexidade extremo e diferentes dimensões. Portanto, solucionar tal questão depende do engajamento coordenado da vasta gama de atores envolvidos, quais sejam: as três esferas administrativas (federal, estadual e municipal); gestores, observadores e beneficiários envolvidos nas diversas ações do plano; o setor empresarial e a sociedade como um todo (pois os hábitos de consumo estão intrinsecamente relacionados ao problema).

O maior desafio, como confirmado pelas avaliações independentes realizadas sobre a implementação do plano e pelas análises de medidas que tiveram maior impacto na redução do desmatamento, está em que sejam alcançados os objetivos de promoção de atividades sustentáveis, pois, apesar de o avanço nos sistemas de monitoramento e ordenamento territorial ter impactos positivos significativos no combate à degradação florestal, atividades econômicas desenvolvidas dentro de um paradigma que exclui a responsabilidade socioambiental apresentam-se como ameaça real à frágil dinâmica de mudança dos solos na região amazônica.

No entanto, promover atividades sustentáveis que garantam a manutenção de baixas taxas de desmatamento em tal região não deve ser resultado apenas da implementação das estratégias do PPCDAm, mas sim da real articulação entre as diretrizes estratégicas desse Plano e políticas públicas como o Programa Plurianual e demais projetos desenvolvimentistas, além da expansão e fortalecimento do Plano Amazônia Sustentável, do Programa de Agricultura de Baixo Carbono, dos Pactos Setoriais com o setor empresarial e de iniciativas de incentivo nutridas pelo veio da sustentabilidade.

68 PRODES, op. cit.

69 Ibidem. 


\section{SOBRE OS AUTORES}

NATÁLIA GIRÃO RODRIGUES DE MELLO é mestranda no Programa de Pós-Graduação em Ciência Ambiental (Procam) do Instituto de Energia e Ambiente da Universidade de São Paulo (IEE/USP).

E-mail: nataliagrdemello@usp.br

PAULO ARTAXO é professor doutor no Instituto de Física da Universidade de São Paulo (IF/USP).

E-mail: artaxo@if.usp.br

\section{REFERÊNCIAS BIBLIOGRÁFICAS}

ABDALA, Guilherme. Plano de Ação para Prevenção e Controle do Desmatamento na Amazônia Legal (PPCDAm) Documento de avaliação 2004-2007. Brasília, DF: Ministério do Meio Ambiente, 2008.

ALVES, Diogenes et al. The changing rates and patterns of deforestation and land use in Brazilian Amazonia. In: KELLER, M. et al. (Ed.). Amazonia and global change. Washington, DC: AGU, 2009. p. II-23. (Geophysics Monograph Series, I86.)

ARIMA, Eugênio et al. Public policies can reduce tropical deforestation: lessons and challenges from Brazil. Land Use Policy, v. 4I, p. 465-473, 2014.

ASSUNÇÃO, Juliano; GANDOUR, Clarissa; ROCHA, Romero. DETERring deforestation in the Brazilian Amazon: environmental monitoring and law enforcement. Núcleo de Avaliação de Políticas Climáticas da Pontifícia Universidade Católica do Rio de Janeiro (NAPC/PUC-Rio) \& Climate Policy Initiative Rio de Janeiro (CPI Rio), 20I3.

CAPELLA, Ana Cláudia. Perspectivas teóricas sobre o processo de formulação de políticas públicas. Revista Brasileira de Informação Bibliográfica em Ciências Sociais - BIB, v. 6I, p. 25-52, 2006.

DAVIDSON, Eric et al. The Amazon basin in transition. Nature, v. 48I, p. 32I-328, 2012.

FEARNSIDE, Philip. Desmatamento na Amazônia: dinâmica, impactos e controle. Acta Amazonica, v. 36, n. 3, p. 395-400, 2006.

FIELD, Christopher et al. Primary production of the biosphere: integrating terrestrial and oceanic components. Science, v. 28I, n. 237, I998.

HECHT, Susanna. From eco-catastrophe to zero deforestation? Interdisciplinarities, politics, environmentalisms and reduced clearing in Amazonia. Envir. Conserv., v. 39, n. oI, p. 4-I9, 2011.

IPCC - Intergovernmental Panel on Climate Change. Summary for policymakers. In: METZ, B. et al. (Ed.). Climate Change 2007 Mitigation. Contribution of Working Group III to the Fourth Assessment Report of the Intergovernmental Panel on Climate Change. Cambridge: Cambridge University Press, United Kingdom and New York, NY, USA, 2007.

IPEA; GIZ; CEPAL. Avaliação do Plano de Ação para Prevenção e Controle do Desmatamento na Amazônia Legal: PPCDAm 2007-20Io. Brasília: Ipea; GIZ; Cepal, 20II. Disponível em: <http://repositorio.cepal. 
org/bitstream/handle/II362/3046/S33375A94520II_pt.pdf;jsessionid=489F2CI4D46I7C7B89I4ABEF88C6CooI?sequence=I>. Acesso em: Io maio 2016.

LAURANCE, William et al. Environment: the future of the Brazilian Amazon. Science, v. 29I, n. 5503, p. 438-439, 2001.

MALHI, Yadvinder et al. Climate change, deforestation, and the fate of the Amazon. Science, v. 3I9, n. 5860, p. I69-I72, 2008.

BRASIL. Ministério do Meio Ambiente - MMA \& Serviço Florestal Brasileiro - SFB. Florestas do Brasil em resumo (Relatório). Dados de 2007-20I2. Brasília, DF: MMA e SFB, 2013.

MORTON, Douglas; SALES, Márcio; SOUZA, Carlos; GRISCOM, Bronson. Historic emissions from deforestation and forest degradation in Mato Grosso, Brazil: I) source data uncertainties. Carbon Balance and Management, 6:I8, 20 II.

NEPSTAD, Daniel; STICKLER, Claudia; ALMEIDA, Oriana. Globalization of the Amazon soy and beef industries: opportunities for conservation. Conservation Biology, v. 20, n. 6, p. I595-I603, 2006.

NEPSTAD, Daniel et al. Interactions among Amazon land use, forests and climate: prospects for a nearterm forest tipping point. Philosophical Transactions of the Royal Society B: Biological Sciences, v. 363, n. I498, p. I737-I746, 2008.

PPCDAm. Plano de Ação para Prevenção e Controle do Desmatamento da Amazônia Legal. Fase I. Brasília, DF: Casa Civil, 2004.

. Fase II. Brasília, DF: Casa Civil, 2009.

. Fase III. Brasília, DF: Casa Civil, 2012.

PRATES, Rodolfo Coelho. O desmatamento desigual da Amazônia brasileira: sua evolução, suas causas e consequências sobre o bem-estar. Tese (Doutorado em Ciências). Escola Superior de Agricultura "Luiz de Queiroz”, Universidade de São Paulo, Piracicaba, 2008.

PRODES. Programa de Monitoramento da Floresta Amazônica Brasileira por Satélite. Ministério do Meio Ambiente - MMA, Instituto Nacional de Pesquisas Espaciais - Inpe do Ministério da Ciência e Tecnologia. Disponível em: 〈http://www.obt.inpe.br/prodes >. Acesso em: I9 dez. 20I4.

RAJÃO, Raoni; VURDUBAKIS, Theo. On the pragmatics of inscription: detecting deforestation in the Brazilian Amazon. Theory, Culture er Society, v. 30, n. 4, p. I5I-I77, 2013.

RIVERO, Sérgio; ALMEIDA, Oriana; ÁVILA, Saulo; OLIVEIRA, Wesley. Pecuária e desmatamento: uma análise das principais causas diretas do desmatamento na Amazônia. Nova Economia, v. I9, n. I, p. 4I-66, 2009.

SETZER, Alberto et al. O caso de um desmate ilegal na Amazônia antecipado pela detecção de fogo e da degradação ambiental. In: SenGeF - SEMINÁRIO DE ATUALIZAÇÃO EM SENSORIAMENTO REMOTO E SISTEMAS DE INFORMAÇÕES GEOGRÁFICAS APLICADOS Ã ENGENHARIA FLORESTAL, IO., Curitiba, 20I2. Disponível em: <http://queimadas.cptec.inpe.br/ rqueimadas/documentos/20I2IO_ Setzer_etal_DesmateIlegal_XSengef.pdf $>$. Acesso em: Io maio 2016.

SOARES-FILHO, Britaldo et al. Modelling conservation in the Amazon basin. Nature, v. 440, p. 520- 523, 2006.

SOARES-FILHO, Britaldo et al. Role of Brazilian Amazon protected areas in climate change mitigation. Proceedings of the National Academy of Sciences, v. I07, n. 24, p. I082I-I0826, 2010.

SOUZA, Celina. Políticas públicas: uma revisão da literatura. Sociologias, v. 8, n. I6, 20-45, 2006. 